\title{
Cholesterol crystal embolisation to the alimentary
}

\section{tract}

\author{
W Moolenaar, C B H W Lamers
}

\begin{abstract}
The features of cholesterol crystal embolisation (CCE) to the alimentary tract were studied by retrospective analysis of the clinical and pathological data of 96 patients ( 70 men, 26 women, mean age 73.8 (58-95) years) with this diagnosis in the Dutch national pathology information system (Pathologisch Anatomisch Landelijk Geautomatiseerd Archief (PALGA)) from 1973-92. In the 96 patients, 130 CCE sites were found throughout the alimentary tract, mostly in the colon $(42 \cdot 3 \%)$. Most patients had a history of atherosclerotic disease and presented with abdominal pain, diarrhoea, or gastrointestinal bleeding, sometimes after surgical or radiological vascular procedures. A number were taking oral anticoagulant treatment. The diagnosis of CCE had been considered before the histological diagnosis in only 11 patients. In the remaining cases, ischaemic colitis, tumour, and inflammatory bowel disease were suggested in the differential diagnosis. A premortem diagnosis of CCE was made in $70.8 \%$ of the cases. In 24 of the 35 necropsy examinations, CCE seemed to be directly or indirectly related to the cause of death. It is concluded that in this unselected, homogenous group of patients, CCE sites were most frequently found in the colon. They generally presented with abdominal pain, diarrhoea, and gastrointestinal blood loss. CCE often mimicked common gastrointestinal disease, leading to incorrect diagnosis.

(Gut 1996; 38: 196-200)
\end{abstract}

Keywords: cholesterol crystal, embolisation, alimentary tract, gastrointestinal tract.

Department of Internal Medicine, Wilhelmina Hospital, Assen, The

Netherlands

W Moolenaar

Department of Gastroenterology and Hepatology, University Hospital, Leiden, The Netherlands C B H W Lamers

Correspondence to: Dr W Moolenaar, Department of Internal Medicine, Wilhelmina Hospital, Postbus 30001, 9400 RA Assen, The Netherlands

Accepted for publication 15 August 1995
The phenomenon of cholesterol crystal embolisation (CCE) is defined as dislodgement of separate cholesterol crystals $(<200 \mu \mathrm{m})$, as opposed to large atheromatous particles, $(>200 \mu \mathrm{m})$, from atheromatous plaques, after erosion of their intimal surfaces. These crystals can occlude downstream arterioles. A thrombus subsequently forms and organises around this embolus. ${ }^{1}$ The blood clot is removed, but the cholesterol crystals remain and are encased by intimal tissue and foreign body giant cells. Recanalisation of the thrombus takes place between or beside the crystals, forming slit like vascular spaces. In a completely organised lesion the artery is occluded by cholesterol crystals, often surrounded by foreign body giant cells, slit like vascular spaces, and hyperplastic intimal tissue.
This is the general picture of CCE in histological sections of biopsy specimens, of surgical specimens, and of post mortem tissue, in all of which both more and less advanced lesions can be seen at the same time. The cholesterol crystals are dissolved during the histotechnical procedure, leaving the pathognomonic needle shaped lacunae in the lumina of arterioles in the histological sections (Fig 1).

The disseminated or local obstruction of small vessels gives rise to a highly variable clinical presentation due to the ensuing ischaemia, infarction, possible perforation, or repair reaction. ${ }^{2}$ The effects of CCE range in severity from transient local ischaemia of the skin to failure of multiple organs in the multiple cholesterol emboli syndrome. In necropsy studies the prevalence of CCE ranges from $3 \%{ }^{3}$ to $17 \cdot 6 \%{ }^{4}$

After we had initially misdiagnosed a patient with CCE to the large bowel and gall bladder as having Crohn's disease with marantic acalculous cholecystitis, ${ }^{5}$ we reviewed the published reports on the clinical presentation of gastrointestinal CCE. ${ }^{6}$ In this study we analysed the features of the patients with CCE to the alimentary tract recorded in the Dutch national pathology information system (PALGA) from 1973-92.

\section{Methods}

In 1971, Dutch pathologists founded a computerised database for pathological anatomical reports in The Netherlands, known as PALGA. This network gradually expanded until in 1989 all 70 Dutch pathology departments were participating. Thus PALGA became a permanent body in Dutch health care and a unique source of pathological anatomical information covering a population of 15 million. Standardised abstracts from

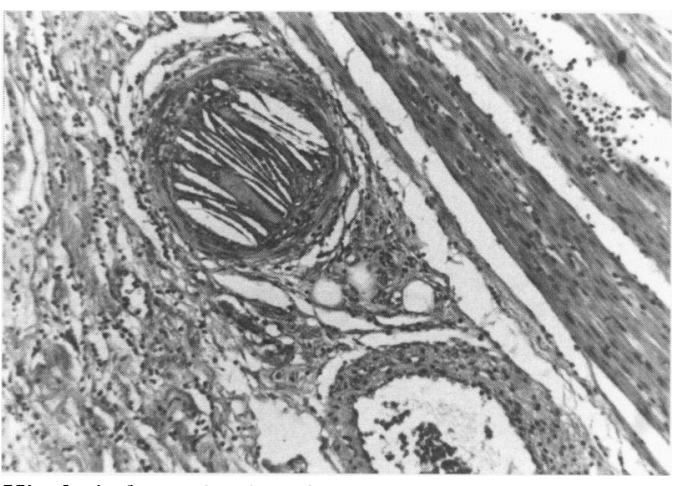

Histological examination of the colon wall showing the typical needle shaped clefts in the lumen of a submucosal arteriole, corresponding to cholesterol crystals, dissolved during the histotechnical procedure. (Original magnification $\times 100$ ). 
TABLE I Distribution of 130 cholesterol crystal embolisation sites in the alimentary tract of 96 patients

\begin{tabular}{lc}
\hline Site & No (\%) \\
\hline Oesophagus & $2(1 \cdot 5)$ \\
Stomach & $16(12 \cdot 3)$ \\
Small bowel & $43(33 \cdot 0)$ \\
Colon & $55(42 \cdot 3)$ \\
Appendix & $1(0 \cdot 8)$ \\
Rectum & $12(9 \cdot 2)$ \\
Anus & $1(0 \cdot 8)$ \\
Total & $130(100)$ \\
\hline
\end{tabular}

local clinical and pathological data (maximum of 360 characters) and diagnoses (maximum of 1000 characters) are submitted for each patient at regular, daily intervals, according to the Systmatized Nomenclature of Medicine (SNOMED) and the Systematized Nomenclature of Pathology (SNOP). Within this data reduction process the patient's privacy is protected by encryption of vital items like name and date of birth. A scientific council has to approve each search before it is to be executed.

Our search was directed to all patients filed with a histologically proved diagnosis of CCE to the alimentary tract between January 11973 and December 31 1992. Subsequently, the respective pathologists were asked by PALGA to issue (through PALGA) the unabbreviated versions of the pathological, anatomical, and clinical reports, after erasing the names of the patients. Privacy protection rules prevented us from obtaining full reports on all patients, since we were not allowed to contact pathologists, clinicians, or hospital departments directly to ask for further information.

From the material gathered all pertinent data regarding demography, history, provocative factors, clinical presentation, CCE sites, pathological anatomy, source of tissue, extraintestinal CCE sites, clinical differential diagnosis, applied therapy irrespective of diagnosis, outcome, and general data source were entered by the first author into a computerised data base, from which the following results were extracted.

\section{Results}

We reviewed the pathological, anatomical, and clinical data of 96 patients ( 70 men, 26 women, mean age 73.6 years, distributed between 58 and 95 years), with a histologically proved diagnosis of CCE to a total of 130 sites throughout the alimentary tract (Table I). The histological diagnosis was made on tissue from 46 resections, 35 necropsy examinations, and 24 biopsies. In two patients, CCE was seen in biopsy specimens as well as in subsequent resected tissues. In another two, CCE was seen both in biopsy specimens and in material from subsequent necropsy examinations, and in five subjects in both resected tissues and in material from necropsy examination. The maximum interval between the first and second report of CCE in these nine patients was two months. The seven patients who underwent necropsy examination died within one month of the first report of CCE.
Abstracts from PALGA along with full pathological-anatomical reports were obtained for 40 of the 96 patients $(41 \cdot 7 \%)$. In 31 patients $(32.3 \%)$, a full clinical report was received as well, but in 25 patients $(26.0 \%)$ only a PALGA abstract was available. Forty seven $(49 \%)$ patients were reported between 1990 and 1992, while none were reported before 1982 . In 35 patients $(36.5 \%)$ no previous history was specified. Of the remaining 61 patients, 15 were known to have had hypertension, 13 had had a myocardial infarction, 11 had signs of coronary insufficiency, nine had intermittent claudication, eight had an aortabifurcation prosthesis, four suffered from diabetes mellitus, four had undergone an episode of ischaemic colitis, and three had earlier had blue toes.

In 59 patients $(61.4 \%)$ no possibly provocative factors were mentioned. Among the remaining 37, 15 were taking oral anticoagulants, 15 had undergone aortic angiography, 12 had had surgery for rupture of an abdominal aneurysm, four had received coronary artery bypass grafts, and one had had thrombolytic therapy, all within eight weeks of the symptoms of CCE, These presenting symptoms are listed in Table II.

Of 12 patients in whom differentiation of white blood cells was reported, one showed eosinophilia of $15 \%$.

Three of the eight patients in whom total serum cholesterol was reported had slightly raised values $(7 \cdot 0,7 \cdot 3$, and $8.0 \mathrm{mmol} / \mathrm{l})$. Complement status was determined in one patient, and reported to be normal. All other reported laboratory findings were non-specific representatives of tissue damage and inflammation.

The diagnoses put forward by the respective clinicians as the most probable in advance of the histological confirmation of the presence of CCE, are listed in Table III.

Specifications of the endoscopic and pathological appearance at the CCE site are listed in Table IV. No CCE sites outside the alimentary tract were mentioned in the reports of 63 patients $(65 \cdot 6 \%)$. In the other 33 patients, 77 sites were located, distributed over the kidneys $(n=25)$, the spleen $(n=12)$, the skin $(n=9)$,

TABLE II Distribution of presenting clinical signs and symptoms in 71 patients with cholesterol crystal embolism (CCE) to the alimentary tract. None were specified in the remaining 25 patients

\begin{tabular}{lc}
\hline Symptom & No (\%) \\
\hline Abdominal pain & $24(33 \cdot 8)$ \\
Rectal bleeding & $18(25 \cdot 4)$ \\
Renal failure & $15(21 \cdot 1)$ \\
Diarrhoea & $9(12 \cdot 7)$ \\
Haematemesis/melaena & $9(12 \cdot 7)$ \\
Anaemia/occult blood loss & $7(9 \cdot 9)$ \\
Livedo reticularis & $6(8 \cdot 5)$ \\
Blue toe(s) & $5(7 \cdot 0)$ \\
Septic shock & $4(5 \cdot 6)$ \\
Weight loss & $4(5 \cdot 6)$ \\
Acalculous cholecystitis & $2(2 \cdot 8)$ \\
Fistula & $2(2 \cdot 8)$ \\
Intra-abdominal bleeding & $2(2 \cdot 8)$ \\
Anal tumour & $1(1 \cdot 4)$ \\
Dyspepsia & $1(1 \cdot 4)$ \\
CCE in fundo & $1(1 \cdot 4)$ \\
Ileus & $1(1 \cdot 4)$ \\
Pancreatitis & $1(1 \cdot 4)$ \\
\hline
\end{tabular}


TABLE III Diagnoses made before histological examination in 70 patients with cholesterol crystal embolisation (CCE) to the alimentary tract. Among these, four cases of malignancy were histologically confirmed as well as two cases of radiation enteritis. All other diagnoses were eventually dismissed and replaced by CCE only. None were put forward in the remaining 26 patients

\begin{tabular}{lc}
\hline Diagnosis & No (\%) \\
\hline Of unknown cause & $12(17 \cdot 1)$ \\
CCE & $11(15 \cdot 7)$ \\
Ischaemic colitis & $9(12 \cdot 9)$ \\
Malignancy & $8(11 \cdot 4)$ \\
Ruptured aneurysm & $4(5 \cdot 7)$ \\
Mesenterical thrombosis & $3(4 \cdot 3)$ \\
Crohn's disease & $3(4 \cdot 3)$ \\
Amyloid & $2(2 \cdot 9)$ \\
Diverticulitis & $2(2 \cdot 9)$ \\
Angiodysplasia & $2(2 \cdot 9)$ \\
Vasculitis & $2(2 \cdot 9)$ \\
Radiation enteritis & $2(2 \cdot 9)$ \\
Peptic ulcer & $2(2 \cdot 9)$ \\
Amoebiasis & $1(1 \cdot 4)$ \\
Primary fistula & $1(1 \cdot 4)$ \\
Ulcerative colitis & $1(1 \cdot 4)$ \\
Rectal polyp & $1(1 \cdot 4)$ \\
Periarteritis nodosa & $1(1 \cdot 4)$ \\
Ischaemic enteritis & $1(1 \cdot 4)$ \\
Appendicitis & $1(1 \cdot 4)$ \\
\hline
\end{tabular}

the liver $(n=7)$, the pancreas $(n=7)$, the adrenals $(n=3)$, the prostate $(n=3)$, the urinary bladder $(n=3)$, the gall bladder $(n=2)$, the muscles $(n=2)$, the brain $(n=2)$, the eye $(n=1)$, and the uterus $(n=1)$.

No specific treatment in relation to CCE was described in 14 patients $(14.6 \%)$. Of the remaining 82 patients, 48 were treated surgically by resection of the damaged tissue and 34 received only supportive care. Of the 40 patients known to have died $(41 \cdot 6 \%), 35$ underwent necropsy. Seven patients had died of septic shock after bowel perforation at the CCE site, eight of multiple organ failure caused by the multiple cholesterol emboli syndrome, four of ongoing blood loss from the CCE sites, three of CCE induced renal failure, one of pancreatitis, and one of diffuse intestinal necrosis both caused by CCE. In the remaining 11 , a cause of death unrelated to CCE was identified.

\section{Discussion}

CCE occurs when cholesterol crystals dislodged from eroded large vessel atheromas are taken downstream and enter the microcirculation. This occurs either spontaneously ${ }^{4}$ or after pharmacological $^{78}$ or mechanical ${ }^{9} 10$ provocation. The position of the nidus, the amount of launched crystals, and the allotment of the bloodstream, along with its capacity for collateral formation will determine the damage done to a particular organ once arterioles are blocked. A study of collected cases ${ }^{11}$ showed the skin and the kidneys to be the most common targets of CCE, along with the spleen and the pancreas. Next came involvement of the alimentary tract - this was present in $9.1 \%$ of the necropsy cases in whom CCE was found.

Blood loss was the most frequently occurring gastrointestinal symptom. The anatomy of the arterial supply, particularly that of the intra-abdominal alimentary tract by way of three major unpaired arteries, should make the organ an easy target for CCE. Furthermore, taking into account that the collateral supply may be only marginally adequate in certain areas, we would expect to find a considerable incidence of clinically relevant gastrointestinal CCE, especially in the elderly atherosclerotic population, waylayed by ever more sophisticated pharmacological, radiological, and surgical threats to atheroma integrity. To place the condition in perspective we compared the figures pertaining to CCE derived from PALGA with those from the Dutch national medical registry (Landelijke Medische Registratie (LMR)). The LMR records every diagnosis for which patients were admitted to Dutch hospitals according to the International Classification of Diseases, 9th Revision, Clinical Modification (ICD-9-CM). Since 1989 PALGA and LMR have filed every histology and autopsy report generated by all 70 Dutch pathology laboratories, and every admission diagnosis to all 157 Dutch hospitals. Both are thus covering a population of 15 million.

From 1989-92, 1494 patients with clinically diagnosed thromboembolic infarctions of the alimentary tract were admitted to Dutch hospitals, ${ }^{12} 1146$ of whom were recorded by PALGA to have a pathological diagnosis of ischaemic colitis. In $37(3 \cdot 2 \%)$ of these cases CCE was given as the most probable cause. Furthermore, over this period 2714 patients were seen with ulcers, perforations, and/or fistulas in the alimentary tract. In 20 of these patients $(0 \cdot 74 \%)$ CCE was the given explanation. Of the 8495 patients who presented to Dutch hospitals between 1989 and 1992 with gastrointestinal bleeding, we documented (through PALGA) $17(0 \cdot 2 \%)$ patients with gastrointestinal CCE. Sixty one of the 4685 cases $(1 \cdot 3 \%)$ of renal failure, admitted to hospital between 1989 and 1992 could be attributed to CCE. From 1972-92 renal insufficiency caused by CCE was reported in $17 \cdot 8 \%$ of all cases in which CCE was present, whereas the frequency of intestinal ischaemia due to CCE in these reports was $5 \cdot 5 \%$.

Except for ischaemic colitis no specific pathological diagnoses filed in PALGA could be connected with the clinical diagnoses mentioned. From 1989-92, CCE to the alimentary tract was found at necropsy in only 17 patients.

TABLE IV Pathological anatomy at the cholesterol crystal embolisation (CCE) site as described in 100 out of all 105 pathological anatomical reports on 96 patients with CCE to the alimentary tract

Necrosis

Ischaemic inflammation

Ulceration

Perforation

Pseudopolyps

Granuloma

Purple spots

Erosions

Haemorrhagic gastritis

Regeneration

Stenosis

Carcinom

Fibrosis

Radiation stigmata

Diverticulitis

Duodenal ulcer

Oesophagitis 
With a mean of 129850 deaths per year and a mean necropsy rate of $7 \cdot 1 \%$, this would point at a frequency of gastrointestinal $\mathrm{CCE}$ of $0.046 \%$. With regard to the prevalence of atherosclerosis and the target positions of both kidney and alimentary tract, as well as the fact that CCE is often a multisystem disease, one might expect a higher incidence of renal CCE and a relatively higher incidence of gastrointestinal CCE. However, all that can be stated at this point is that the figures mentioned can represent either a low incidence or lack of awareness of clinically relevant CCE.

Addressing the latter issue we set out to increase our familiarity with the phenomenon by investigating the ways in which CCE to the alimentary tract manifests itself. We did this by reviewing unselected cases as they were uniformly filed in the national database. The finding of an age and sex distribution similar to that in patients with peripheral arteriosclerotic disease as a whole ${ }^{13}$ agrees with the suggested pathophysiology of CCE. ${ }^{1}$ The distribution of CCE sites in the alimentary tract (Table I) more or less represents the distribution of blood flow once it has passed the atheromatous large vessels, picking up the cholesterol crystals on its way. Several case reports described CCE to the stomach ${ }^{14-17}$ the small intestine, ${ }^{16-21}$ and the colon. ${ }^{62-28}$ To our knowledge there have been no published reports of oesophageal CCE. The emboli, probably originating from the coeliac trunk, taken downstream by the left gastric artery and its oesophageal branches, caused an ulcer in one patient, and a chronic refractory oesophagitis in another. Other as yet unreported sites are the appendix and the anus, for both of which we found one example - the former in the shape of a granulomatous appendicitis, the latter leading to the formation of an anal ridge initially believed to be a malignant tumour.

In concordance with the pathophysiology and with earlier reports most patients had a history of atherosclerotic disease. In $38.6 \%$ of the reports we studied, a provocative factor could be identified, mostly oral anticoagulant treatment and aortic angiography. The classic clinical presentation emerging from our study is that of a patient with sudden abdominal pain, diarrhoea, gastrointestinal blood loss, renal failure, and skin abnormalities. This fullblown picture was mainly seen shortly after vascular manipulation when massive cholesterol crystal showers must have harassed the downstream tissues. Most patients, however, presented with isolated, often fluctuating, common gastrointestinal symptoms like a dull abdominal pain, dyspepsia, chronic anaemia due to occult blood loss, weight loss, a palpable mass, fistula, or ileus (Table II). This is probably a result of spontaneous, chronic, intermittent cholesterol crystal scattering, giving rise to mucosal dysfunction or damage, which may be transient.

In our study a diagnosis of CCE before the histological examination was made in only 11 patients (Table III). Another reason for this, beside a low index of suspicion or the presence of another disease explaining the symptoms, may be that the pathognomonic needle shaped clefts in the deeper submucosal arteries are either easily overlooked in histological sections or missed in superficial biopsy specimens. ${ }^{22}$ An early diagnosis was greatly facilitated by the presence of preceding or concomitant CCE manifestations in skin or kidneys.

We registered many gastro-intestinal diseases as later dismissed differential diagnoses (Table III). Some of these were reported earlier, like ischaemic colitis due to other causes, ${ }^{29}$ malignancy, ${ }^{27}$ Crohn's disease, ${ }^{5}$ angiodysplasia, ${ }^{15}$ vasculitis, ${ }^{30}$ periarteritis nodosa, ${ }^{31}$ and rectal polyp. ${ }^{22}$ Much of this is due to the observed stages of mucosal damage and repair as well as granuloma formation (Table IV), that may lead the clinician further astray when the CCE clefts go unnoticed or escape superficial sampling. The macroscopic lesions such as pseudopolyps, purple spots, erosions, stenosis, ulcers, and telangiectasia were noted at endoscopy, or by observing the resection specimen. Endoscopic photographs of purple discolourations and geographic whitish macules of the mucosa and of sessile polyps, all proved to be caused by CCE, have previously been published. ${ }^{32} 33$

In six patients, another cause (carcinoma in four and radiation effects in two) explained the symptoms, suggesting that CCE was a coincidental finding. Eosinophilia, earlier reported to accompany CCE to the kidneys, ${ }^{34}$ was seen in one of 12 patients in whom a white blood cell count was reported. Similarly, hypocomplementaemia ${ }^{35}$ was not found in the one patient tested. The raised serum cholesterol values found, probably represent a common risk factor for the development of atherosclerotic plaques commonly present in the studied population. The locations of the extraintestinal CCE sites we observed follow the same distribution as described in an earlier study of cases collected from published reports. ${ }^{11}$

Since we only searched for CCE to the alimentary tract, the cases of gall bladder, liver, and pancreas CCE we found were restricted to those in whom these were additional findings. Clinical expressions due to these locations were limited to two cases of acalculous cholecystitis in two patients with gall bladder CCE and one of pancreatitis in seven patients with CCE to the pancreas. The last finding suggests that CCE to the liver and the pancreas can be asymptomatic.

The retrospective nature of our study precludes a reliable statistical analysis of true incidence, treatment, and outcome figures. Answers concerning true incidence, however, will be hard to come by owing to the ethical objections to taking multiple biopsy specimens prospectively from high risk patients. Once the diagnosis of CCE is made the patient can be spared further diagnostic efforts and treatment can be adjusted. Consequent location and exclusion or excision of the emboli source, although earlier described as a seldomly reached objective, ${ }^{36}$ was recently reported to give excellent long term relief of embolisation in a series of 62 patients. ${ }^{37}$ Excision of the damaged tissue may give considerable, albeit 
only temporary relief, due to the often undulant but relentless course of the disease. The latter is illustrated by our finding of necropsy rapidly following biopsies and resections from the same patients.

In conclusion, studying clinical and pathological data of an unselected homogenous group of patients we found that common gastrointestinal symptoms can be caused by CCE. CCE was most seen in the colon. It was also observed as a coincidental finding. Its early diagnosis by prompt and deep biopsies of endoscopically identified lesions or skin biopsy might obviate elaborate diagnostic procedures and advance adequate care.

The authors are indebted to Mrs IJAMG Casparie - van Velsen MD, advisor of the PALGA scientific council, for executing the computer search.

Furthermore, they gratefully acknowledge the cooperation of clinicians and pathologists. A full list of institutions participating in the study is available from the authors.

1 Flory CM. Arterial occlusions produced by emboli from eroded aortic atheromatous plaques. Am $\mathcal{F}$ Clin Pathol 1945; 21: 549-65.

2 Rosman HS, Davis TP, Reddy D, Goldstein S. Cholesterol embolization: clinical findings and implications. $7 \mathrm{Am} \mathrm{Col}$ Cardiol 1990; 15: 1296-9.

3 Cross SS. How common is cholesterol embolism? 7 Clin Pathol 1991; 44: 859-61.

4 Gore I, Collins DP. Spontaneous atheromatous embolization. Am 7 Clin Pathol 1960; 33: 416-26.

5 Moolenaar W, Kreuning J, Elderink F, Lamers CBHW. Ischemic colitis and acalculous cholecystitis as rare manifestations of cholesterolemboli in the same patient. Am $\mathcal{J}$ Gastroenterol 1989; 84: 1421-2.

6 Moolenaar W, Lamers CBHW. Cholesterol crystal embolization and the digestive system. Scand $\mathcal{f}$ Gastroenterol 1991; 26 (Suppl 188): 69-72.

7 Feder W, Auerbach R. "Purple toes": An uncommon sequela of oral coumarin drug therapy. Ann Intern Med 1961; 55: 911-7.

8 Shapiro LS. Cholesterol embolization after treatment with tissue plasminogen activator. $N$ Eng 7 Med 1989; 321: 168-70.

9 Gaines PA Kennedy A Moorhead P, Cumberland CD, Welsh CL, Rutley MS. Cholesterol embolization: a lethal complication of vascular catheterization. Lancet 1988; i: 168-70.

10 Thurlbeck WM, Castleman B. Atheromatous emboli to the kidneys after aortic surgery. $N$ Engl $\mathcal{F}$ Med 1957; 257: 442-7.

11 Fine MK, Kapoor W, Falanga V. Cholesterol crystal embolization: a review of 221 cases in the English literature. Angiology 1987; 38: 769-84.

12 Spïsverteringsziekten in Nederland, 1970-1989. Breuke-len: De Nederlandse Lever Darm Stichting, 1993.

13 Statistisch faarboek 1994. Centraal bureau voor de Statistiek. Gravenhage: CBS, 1994.

14 Forouhar FA Morteza M, Gardner P, Smith N. Cholesterol embolism causing bleeding gastric ulcers. Ann Clin Lab Sci 1988; 18: 260-5.

15 Bank S, Aftalion B, Anfang C, Altman H, Wise L. Acquired angiodysplasia as a cause of gastric hemorrhage: a possible consequence of cholesterol embolization. Am $\boldsymbol{f}$ Gastroenterol 1983; 78: 206-9.

16 Anderson WR, MacDonell Richards A, Weiss $L$. Hemorrhage and necrosis of the stomach and bowel due to atheroembolism. Am f Clin Pathol 1967; 48: 30-8.

17 Taylor NS, Gueft B, Lebowich RJ. Atheromatous embolization: a cause of gastric ulcers and small bowel necrosis. Gastroenterology 1964; 47: 97-103.

18 Smith FCT, Boon A, Shearman CP, Downing $R$. Spontaneous cholesterol embolisation: a rare cause of bowel infaraction. European fournal of Vascular Surgery 1991; 5: 581-2.

19 Blundell JW. Small bowel stricture secondary to multiple cholesterol emboli. Histopathology 1988; 13: 459-62.

20 Socinski MA, Frankel J, Morrow PL, Krawitt EL. Painless diarrhea secondary to intestinal ischemia. Diagnosis of atheromatous emboli by jejunal biopsy. Dig Dis Sci 1984; 29: 674-7.

21 Rushovich AM. Perforation of the jejunum: a complication of atheromatous embolization. Am $\mathcal{F}$ Gastroenterol 1983; 78: $77-82$.

22 Gramlich TL, Hunter SB. Focal polypoid ischemia of the colon: atheroemboli presenting as a colonic polyp. Arch Pathol Lab Med 1994; 118: 308-9.

23 Grant DJ, Sanders DSA, McMurdo MET, Lyall MH. Recurrent anaemia due to ischaemic colonic ulceration caused

24 Bartelink AKM, Jaarsveld BC van, Meyer AM, Cluysenaer OJJ. Acute abdominal haemorrhage due to cholesterol crystal embolization. Clinical Intensive Care 1992; 3: 134-6.

25 O'Briain DS, Jeffers M, Kay EW, Hourihane D. Bleeding due to colorectal atheroembolism. Am f Surg Pathol 1991; 15: $1078-82$.

26 Anderson WR, Braverman T. Colon perforation due to cholesterol embolism. Hum Pathol 1991; 22: 839-41.

27 Chan T, Levine MS, Park Y. Cholesterol embolization as a

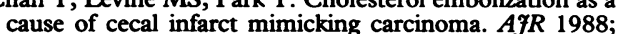
150: $1315-6$.

28 Carnevale NJ, Delany HM. Cholesterol embolization to the cecum with bowel infarction. Arch Surg 1973; 106: 94-6. 29 Robert JH, Mentha G, Rohner A. Ischemic colitis: two distinct patterns of severity. Gut 1993; 34: 4-6.

30 Cappiello RA, Espinoza LR, Adelman H, Aguilar J, Vasey FB, Germain BF. Cholesterol embolism: a pseudovasculitic syndrome. Seminars in Arthritis and Rheumatism 1989; 18: 240-6.

31 Macdonell Richards A, Eliot RS, Kanjuh VI, Bloemendaal RD, Edwards JE. Cholesterol embolism. A multiple f Cardiol 1965; 15: 696-707.

32 Zanen AL, Rietveld AP, Tjen HSLM. Cholesterol embolism: diagnosis by endoscopy. Endoscopy 1994; 26: 257-9.

33 Martin-de-Argila C, Rivera MM, Moreira VV, Redondo C, Otero GGY, Candia A. Duodenoscopic view of cholesterol crystal embolization. Gastrointest Endosc 1994; 40: 371-3.

34 Kasinath BS, Corwin HL, Bidani AK, Korbet SM, Schwartz MM, Lewis EJ. Eosinophilia in the diagnosis of atheroembolic renal disease. Am $f$ Nephrol 1987; 7: 173-7.

35 Cosio FG, Zager RA, Sharma HM. Atheroembolic renal disease causes hypocomplementaemia. Lancet 1985; ii: 118-21.

36 Dahlberg PJ, Frecentese DF, Cogbill TH. Cholesterol embolism: experience with 22 histologically proven cases. Surery 1989; 105: 737-46.

37 Baumann DS, McGraw D, Rubin BG, Allen BT, Anderson CB, Sicard GA. An institutional experience with arterial atheroembolism. Ann Vasc Surg 1984; 8: 258-65. 